\title{
EVALUACIÓN DEL CLIMA ORGANIZACIONAL EN LAS ENTIDADES PRESTADORAS DE SALUD
}

\section{EVALUATION OF ORGANIZATIONAL CLIMATE IN HEALTH PROVIDERS AGENCIES}

\author{
Msc (C). Claudia del Pilar Quintero Prado ${ }^{a}$, Msc. Jose Gregorio Arevalo Ascanio ${ }^{\mathrm{b}}$, Ph.D. Sir \\ Alexci Suarez Castrillon ${ }^{\mathrm{c}}$ \\ ${ }^{a}$ Universidad Francisco de Paula Santander Ocaña, Grupo de Investigación GIDSE, Vía \\ Acolsure Sede el Algodonal, Ocaña - Norte de Santander, Colombia, cpquinterop@ufpsp.edu.co \\ ${ }^{\mathrm{b}}$ Universidad Francisco de Paula Santander Ocaña, Grupo de Investigación GIDSE, Vía \\ Acolsure Sede el Algodonal, Ocaña - Norte de Santander, Colombia, jgarevaloa@ufpso.edu.co \\ ${ }^{c}$ Universidad Francisco de Paula Santander Ocaña, Grupo de Investigación GRUCITE, Vía \\ Acolsure Sede el Algodonal, Ocaña - Norte de Santander, Colombia, sasuarezc@ufpso.edu.co
}

Resumen: Los trabajadores son la base fundamental para el desarrollo y cumplimiento de los objetivos planteados por la organización, en consecuencia sus líderes deben garantizar un ambiente trabajo y un clima organizacional saludable que le permita lograr la efectividad de los procesos y la satisfacción de los clientes. El objetivo general del estudio, fue evaluar el clima organizacional en una entidad prestadora de servicios de salud; para dar cumplimiento a este propósito la investigación se centró en dos ejes: el primero el establecimiento de las percepciones de los trabajadores y los aspectos de motivación laboral a fin de determinar la situación actual de la empresa; en segundo lugar la identificación de las debilidades del clima organizacional a fin de establecer los puntos de mejora. Se realizó un estudio descriptivo soportado con fuentes primarias, una encuesta dirigida a los trabajadores de la organización; así mismo se tuvo en cuenta las teorías relacionadas con el clima organizacional y documentación relevante para la realización de la propuesta.

Palabras clave: organizaciones, clima organizacional, satisfacción en el trabajo, servicio de salud.

\begin{abstract}
Workers are the fundamental basis for the development and fulfillment of the objectives set by the organization, its leaders must therefore ensure work environment and a healthy organizational climate that allows you to achieve the effectiveness of processes and customer satisfaction. The overall objective of the study was to evaluate the organizational
\end{abstract}


climate in a lending institution health services; to fulfill this purpose research focused on two areas: the first establishing perceptions of workers and labor aspects of motivation to determine the current status of the company; secondly identifying the weaknesses of the organizational climate in order to establish the points of improvement. A descriptive study supported with primary sources was conducted a survey of workers in the organization; It also took into account the theories related to organizational climate and relevant to the implementation of the proposal documentation.

Keywords: organizations, organizational climate, job satisfaction, health service.

\section{INTRODUCCIÓN}

La empresa según (Castro , 2005) es un agente importante en la acción económica y laboral, en la distribución de la renta y en la generación de cohesión social. (...) ha de saber que los cambios sociales le afectan y ha de contar con ellos para "reacoplarse" en su reconocimiento social, ésta a su vez está conformada por un grupo de personas que interactúan entre sí y que deben desarrollar actividades de manera conjunta y compartir experiencias en un entorno común que se convierte finalmente en el clima organizacional que prevalece en la organización.

En cuanto al clima organizacional, Schneider y Hall (1972); citados en Santana y Araujo (2007) citados por (Cardona \& Zambrano, 2014) definen el clima organizacional como un conjunto de percepciones que una organización tiene de sus políticas, prácticas y procedimientos y que son compartidos por sus miembros en torno al ambiente laboral.

Lo antes mencionado es confirmado por Gonçalves (2000) ciado por (Mujica \& Pérez, 2007) quien señala el clima organizacional como a las características del medio ambiente de trabajo, las cuales son percibidas directa o indirectamente por los trabajadores que desempeñan en ese medio ambiente y que finalmente repercuten en el comportamiento y cultura laboral.

El presente artículo presenta el diagnóstico del clima organizacional de una entidad prestadora de servicios de salud ubicada en la ciudad de Ocaña Norte de Santander. El estudio se realizó a través de una encuesta con el fin de evaluar seis (6) dimensiones que dieron un total de 25 preguntas.

\section{REVISIÓN DE LITERATURA}

Para el desarrollo del estudio se hizo necesario realizar la identificación de artículos científicos en los temas de Clima Organizacional, teniendo en cuenta artículos, publicados en revistas de alto impacto y en las bases de datos Scopus, Science Direct, Scielo, Emerald y Redalyc. Algunos de los trabajos de investigación encontrados se señalan a continuación:

Inicialmente (Caligore \& Diaz, 2003) asumen la realización de una investigación de carácter descriptiva y de campo para determinar el clima organizacional y el desempeño de los docentes de la ULA, con el propósito de elaborar una propuesta para optimizar la gestión en la institución. Como instrumento para la recolección de 
información acudieron a la elaboración de un cuestionario que contenía una escala de valoración tipo Likert, adicionalmente identificaron unas dimensiones que les permitió obtener resultados importantes para la investigación.

De otro lado (Rodríguez, 2002) en su investigación sobre el clima organizacional en una empresa de telecomunicaciones, toma como referencia el modelo empleado Xavier (1984) quien a su vez referencia la teoría McClelland (1972) sobre la motivación humana y los modelos conceptuales de Kolb (1978) sobre el clima organizacional. Para la investigación el autor seleccionó como muestra los trabajadores de nivel operativo de las áreas de: Dirección de marketing y ventas, consultoría y planificación de marketing, para la recolección de la información utilizó En esta investigación, se utilizó la escala Kolb, inspirada en la obra de Litwin.

Por su parte (Mendez, 2005) presenta los resultados de su investigación sobre el clima organizacional en empresas Colombianas, la cual se fundamentó en una herramienta llamada el IMCOC (instrumento para medir clima en las organizaciones colombianas), Al respecto, la investigación deja claras conclusiones sobre percepciones que tiene los empleados y que son constantes en las organizaciones las cuales corresponden a situaciones que tienen su origen en la aplicación de los modelos administrativos de Taylor y Weber, y en menor proporción la teorías de Fayol, de las relaciones humanas y del comportamiento.

(Salvador, 2012) realiza un estudio con el objetivo de correlacionar el clima organizacional y la satisfacción laboral en personal de salud, utilizando para este propósito métodos como el estudio observacional, transversal, descriptivo, analítico y como herramienta para la recolección de la información se utilizó una escala mixta Likert, como resultado del estudio se concluyó que existe una un clima organizacional favorable el cual redunde en la calidad de vida de los integrantes de la institución de salud y en consecuencia en la calidad del servicio.

Finalmente (Bermúdez, Pedraza , \& Rincón , 2015) en su estudio buscaron realizar una caracterización del clima organizacional desde la mirada de los estudiantes en seis universidades ubicadas en la ciudad de Bogotá. Para lograr su propósito abordaron la metodología desde el enfoque cualitativo, con alcance descriptivo. Consideraron en su estudio que la percepción de los estudiantes es imprescindible para entender a las instituciones como un todo que es afectado por factores externos a ella y así mismo el clima organizacional que se da en la institución educativa, repercute en su experiencia educativa.

\section{METODOLOGÍA}

Tal como lo plantea (Grajales , 2000) La investigación descriptiva, trabaja sobre realidades de hecho y su característica fundamental es la de presentar una interpretación correcta. Esta puede incluir los siguientes tipos de estudios: encuestas, casos, exploratorios, causales, de desarrollo, predictivos, de conjuntos, de correlación. La investigación desarrollada fue de tipo descriptiva y se centró en el enfoque cuantitativo teniendo en cuenta como fenómeno específico "el clima organizacional" 
Por ser una población finita y relativamente pequeña la muestra estuvo conformada por el por el $100 \%$ de los empleados que laboran en la institución para un total de 20 trabajadores.

Como instrumento de medición se tomó como base el modelo propuesto por Chiang, salazar y núñez (2007), quienes miden el clima organizacional en siete dimensiones de las cuales se seleccionaron seis que conformaron el cuestionario definitivo: entorno laboral, información y comunicación, conformidad con el puesto de trabajo, progreso profesional y remuneración, conocimiento de la política, objetivos, misión y visión y por último las relaciones de trabajo. Se utilizó una escala de respuesta tipo Likert conformada por cuatro (4) categorías de respuesta dependiendo del tipo de pregunta (nunca, algunas veces, frecuentemente y siempre) (nulo, regular bueno y excelente).

Los cuestionarios se entregaron a los trabajadores sin especificar un tiempo límite para el diligenciamiento del mismo, lo anterior teniendo en cuenta que en estas entidades de prestación de servicios de salud laboran por turnos.

\section{RESULTADOS}

La encuesta aplicada a los trabajadores de la entidad objeto de estudio, estuvo conformada inicialmente por 28 preguntas, luego de aplicada la prueba piloto se redujo a 25, cada pregunta tenía 4 alternativas de respuestas según la escala tipo Likert, para este caso cada uno de los entrevistados debía seleccionar sólo una respuesta. De acuerdo a esa escala se agruparon las respuestas en las dimensiones descritas a continuación:

\section{Dimensión 1. Entorno Laboral}

Tal como lo plantea (Barrios \& Paravic, 2006) (...) si el entorno laboral en una organización es saludable, se habrá adelantado en la conquista de espacios que permitan el desarrollo y promoción de la salud en el trabajo. Por este motivo es primordial analizar su influencia y la percepción que los trabajadores en toda organización.

$\mathrm{Al}$ respecto del entorno laboral en la EPS los trabajadores en su gran mayoría se encuentra satisfechos con las condiciones de trabajo en la que desempeñan sus funciones lo que es un aspecto favorable teniendo en cuenta que siempre cuentan con los medios disponibles para realizar sus labores, de otra parte el porcentaje de trabajadores que consideran que los directivos se interesan en su bienestar es considerable lo que representa un aspecto bastante positivo para que las labores se desarrollen en perfecta armonía.

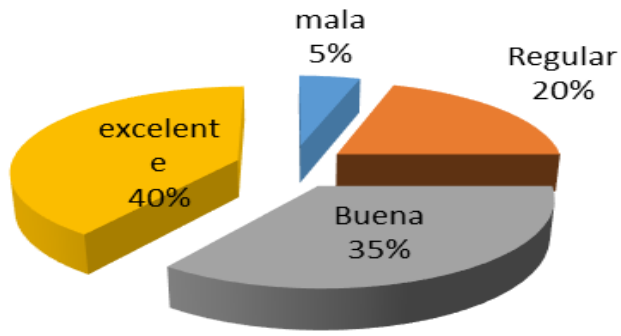

Figura 1. Percepción de los trabajadores sobre el entorno laboral.

\section{Dimensión 2. Información y comunicación}

Al abordar el tema de información y comunicación en la organización es relevante mencionar los estudios de 
Hawthorne citado (Casares, 2007) los cuales dieron a conocer que en el interior de la organización formal de toda empresa actúan grupos informales de trabajo y cuyo buen funcionamiento es sinónimo de satisfacción y productividad. Teniendo en cuenta (...) al clima laboral como un factor más de satisfacción del grupo y de productividad.

Para los trabajadores de la empresa estudiada la información y comunicación al interior de la organización se desarrolla de buena manera, se resaltan aspectos positivos como: el grado en que éstos consideran que están siendo informados de todas las actividades que se desarrollan en la empresa, así mismo coinciden en su gran mayoría en que se les permite realizar sugerencias a los jefes sin temor a que tengan represarias, en un porcentaje alto consideran que sólo algunas veces los directivos consiguen lograr que sus trabajadores trabajen en equipo, un aspecto que debe considerarse para la mejora, teniendo en cuenta que gracias a esta dinámica se puede lograr el cumplimiento de los objetivos eficazmente.

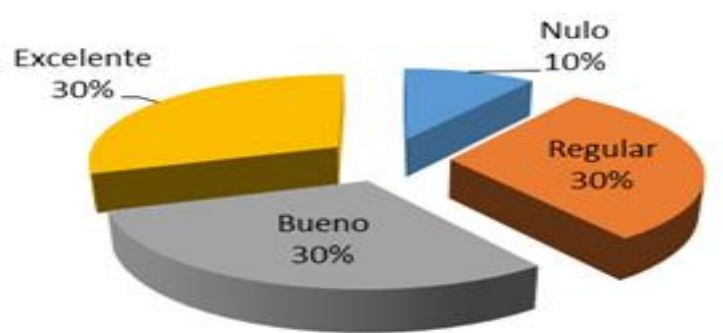

Figura 2. Satisfacción con la información que recibe de la institución.

\section{Dimensión 3. Conformidad con el puesto de trabajo}

El puesto de trabajo en una organización se concibe como todas las actividades que debe desempeñar un trabajador teniendo en cuenta las expectativas de la organización y del equipo de trabajo y la de cada uno de sus miembros.
Teniendo en cuenta lo anterior se pudo determinar en este aparte que en su gran mayoría los trabajadores se encuentran conformes con su puesto de trabajo, se evidencia la coherencia que existe entre la formación y experiencia del personal con el cargo que ocupan. Otro aspecto de resaltar es que a la mayoría del personal le gustaría seguir trabajando en la empresa por mucho más tiempo.

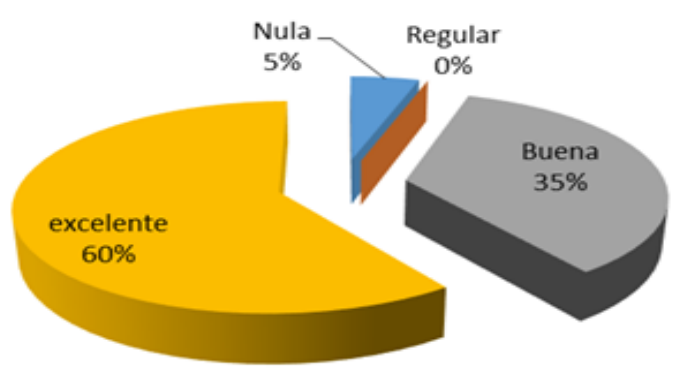

Figura 3. Relación de la experiencia con el puesto de trabajo.

\section{Dimensión 4. Progreso profesional y remuneración}

Con relación al desarrollo profesional (Fernandez , 2002) lo define como el proceso por el que las personas progresan a través de una serie de etapas caracterizadas por distintas tareas de desarrollo, actividades y relaciones.

Para el caso en particular, los trabajadores de la organización manifestaron estar inconformes con el salario que reciben por la labor desempeñada, así como con las pocas oportunidades de ascenso. 


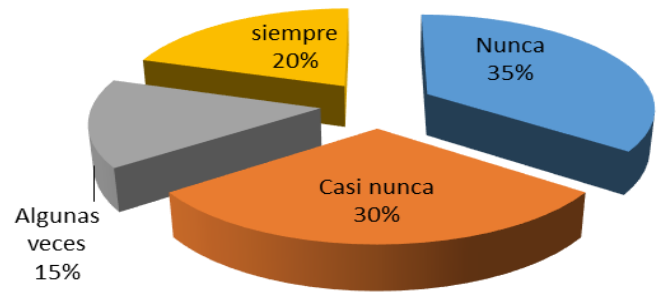

Figura 4. Relación de la experiencia con el puesto de trabajo.

\section{Dimensión 5. Conocimiento de la política, objetivos, misión y visión}

Para toda organización es indispensable la declaración de su misión, visión y el establecimiento de objetivos claros que tracen el funcionamiento de la misma, los cuales deben ser difundidos a todos sus miembros, con la finalidad de orientar las actividades que se realizan en la empresa en una misma dirección.

Sobre este aspecto, los trabajadores tienen opiniones divididas, lo anterior debido a que manifestaron no tener claridad sobre los elementos evaluados (misión, visión, objetivos) pero tienen claridad sobre cómo cada uno aporta con su trabajo al logro de los objetivos planteados en la organización.

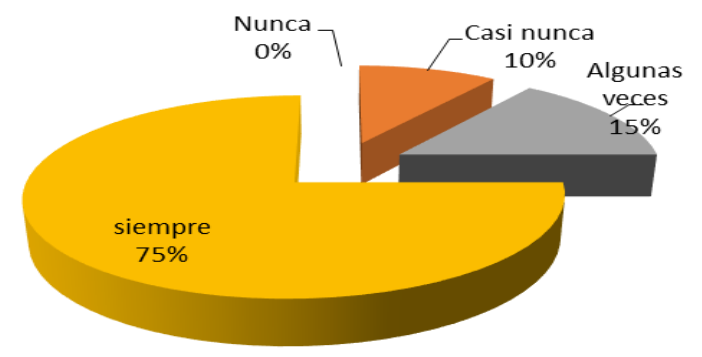

Figura 5. Grado de conocimiento sobre el aporte al cumplimiento de los objetivos.

\section{Dimensión 6. Relaciones de trabajo}

Las relaciones de trabajo influyen en el desempeño de la organización y por ende en la satisfacción de los clientes tanto internos como externos. (Trebilcock, 2001) Define las relaciones de trabajo como (...) el sistema en el que las empresas, los trabajadores y sus representantes, (...) interactúan con el fin de establecer las normas básicas de convivencia.

En último lugar y sin ser la menos importante, existe una percepción positiva sobre las relaciones de trabajo, puesto que en su gran mayoría los encuestados consideran que éstas son excelentes, es probable que se deba a la construcción de relaciones naturales y espontáneas por la afinidad de las personas y no por ser una cultura promovida por los directivos de la empresa.

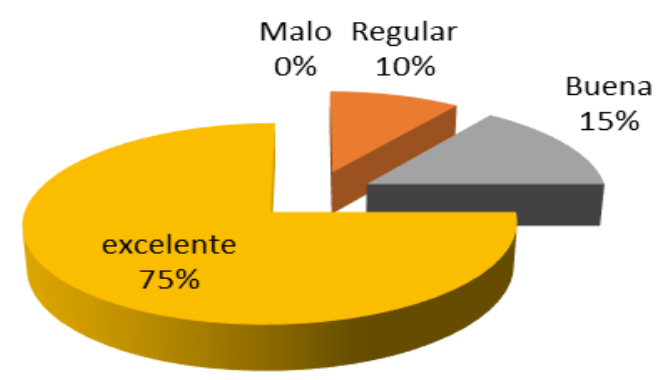

Figura 6. Grado de cooperación entre compañeros de trabajo. 


\section{CONCLUSIONES}

La complejidad del comportamiento humano condiciona, en muchas ocasiones, que las relaciones interpersonales de una institución no se desarrollen en un clima de cordialidad y armonía, lo que influye en el logro de los objetivos, pero este caso no de la entidad prestadora de servicios de salud, ya que si analizamos su contexto de manera general se puede considerar como un resultado satisfactorio para la organización, es indispensable realizar una análisis de manera específica para identificar algunas debilidades que deben ser atendidas y a través de acciones preventivas y un plan de mejora no se ponga en riesgo el clima existente.

Básicamente el estudio ha servido para identificar los aspectos fuertes del clima en la organización, así como las debilidades reales o potenciales resaltando en este caso la inconformidad de los trabajadores en cuanto a la remuneración y a las posibilidades de ascenso, (...) aspectos que según (Carlucci \& Schiuma, 2012) deben ser tenidos en cuenta por la alta dirección con el fin de gestionar mejor los recursos intangibles de la organización.

Otro tema importante de considerar es la necesidad latente de implementar acciones para logar que el trabajo en equipo se realice de manera más frecuente, más aún cuando fomentar esta práctica le permite a toda organización obtener más y mejores resultados.

Finalmente y dadas las dimensiones del clima organizacional, es importante que las directivas de la organización comprendan las implicaciones que éste tema tiene no sólo en la salud de los trabajadores si no, en la efectividad de la organización, razón por la cual debe ser tenido en cuenta en el proceso administrativo y más aún en la toma de decisiones estratégicas; por consiguiente son los líderes los responsables de lograr la integración de todos los elementos que la conforman y se requiere la actualización de sus conocimientos en cuanto a nuevas técnicas para la gestión humana

\section{BIBLIOGRAFÍA}

Barrios, S., \& Paravic, T. (2006). Promoción de la salud y un entorno laboral saludable . Latino-am efermagem, 36-41.

Bermúdez , J., Pedraza , A., \& Rincón , C. (2015). El Clima Organizacional en universidades de Bogotá, desde la perspectiva de los etudiantes. Revista Electrónica de Investigación Educativa, 1-12.

Caligore, I., \& Diaz, J. (2003). Clima organizacional y desempeño de los docentes de la ULA: Estudio de caso. Revista Venezolana de Gerencia, 8(24), 644-658.

Cardona, D., \& Zambrano, R. (2014). Revisión de instrumentos de evaluación de clima organizacional. Estudios Gerenciales , 184-189.

Carlucci, D., \& Schiuma, G. (2012). Evaluating organisational climate through IC lens. Measuring Business Excellenc, 79-90.

Casares, E. (2007). Razón y Palabra. Obtenido

de 
http://www.razonypalabra.org.mx/an

teriores/n56/ecasares.html

Castro , M. (2005). La Responsabilidad social en las empresas, o un nuevo concepto de empresa. CIRIEC, 2951.

Fernandez , N. (2002). El desarrollo profesional de los trabajadores como ventaja competitiva de las empresas. Cuandernos de GEstión, 2, 65-90.

Grajales

$\mathrm{T}$. (2000).

http://tgrajales.net/investipos.pdf.

Obtenido de http://tgrajales.net/investipos.pdf

Kamal , J., Manjit, S., \& See, G. (2015). Organizational climate, trust and knowledge sharing:. Asia Business Studies, 54-77.

Mendez, C. (2005). Clima Organizacional en Empresas Colombianas. Universidad y Empresa, 100-121.

Mujica, M., \& Pérez, I. (2007). Gestión del clima organizacional: Una acción deseable de la Universidad. Laurus, 290-304.

Rodríguez, F. (2002). Clima organizacional: Um estudo em uma empresa de telecomunicacoes. RAE-REvista de administracao de empresas, 42(2), 95-103.

Salgado , J., Remeserio, C., \& Iglesias , M. (1996). Clima organizacional laboral en una PYME. Psicothema, 329-335.

Salvador, A. (2012). Clima organizacional y satisfacción laboral. Revista médica del instituto Mexicano del Seguro Social, 307-314.

Trebilcock, A. (2001). Relaciones laborales y gestión de recursos humanos. En O. i. trabajo, Enciclopedia de salud y seguridad en el trabajo .

Vásquez, R., \& Guadarrama, J. (2001). El clima organizacional en una institución tecnológica de educación superior. Tiempo de Educar, 3(5), 105-131. 